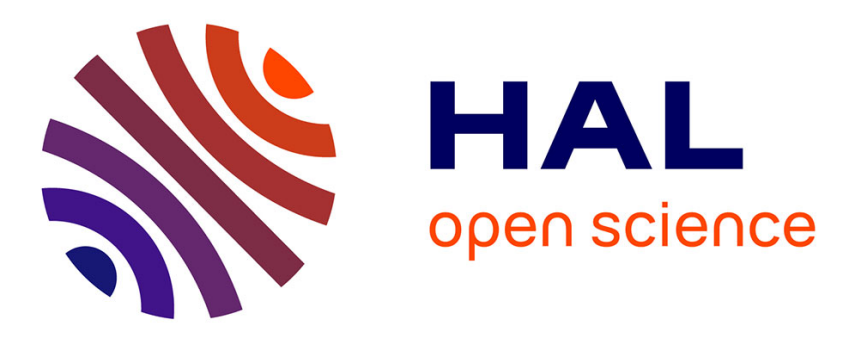

\title{
Lensfree diffractive tomography for the imaging of 3D cell cultures
}

Fabien Momey, Anthony Berdeu, T Bordy, J.-M Dinten, F Kermarrec Marcel, N Picollet-D 'Hahan, X Gidrol, C Allier

\section{- To cite this version:}

Fabien Momey, Anthony Berdeu, T Bordy, J.-M Dinten, F Kermarrec Marcel, et al.. Lensfree diffractive tomography for the imaging of 3D cell cultures. Biomedical optics express, 2016, 10.1364/BOE.7.000949.v008 . inserm-01323080

\section{HAL Id: inserm-01323080 https://www.hal.inserm.fr/inserm-01323080}

Submitted on 30 May 2016

HAL is a multi-disciplinary open access archive for the deposit and dissemination of scientific research documents, whether they are published or not. The documents may come from teaching and research institutions in France or abroad, or from public or private research centers.
L'archive ouverte pluridisciplinaire HAL, est destinée au dépôt et à la diffusion de documents scientifiques de niveau recherche, publiés ou non, émanant des établissements d'enseignement et de recherche français ou étrangers, des laboratoires publics ou privés. 


\title{
Lensfree diffractive tomography for the imaging of 3D cell cultures
}

\author{
F. Momey, ${ }^{1,2, *}$ A. Berdeu, ${ }^{1,2, *}$ T. Bordy, ${ }^{1,2}$ J.-M. Dinten, ${ }^{1,2}$ \\ F. Kermarrec Marcel, ${ }^{1,3,4}$ N. Picollet-D'hahan, ${ }^{1,3,4}$ X. Gidrol, ${ }^{1,3,4}$ and \\ C. Allier ${ }^{1,2}$ \\ ${ }^{1}$ Univ. Grenoble Alpes, F-38000 Grenoble, France \\ ${ }^{2}$ CEA, LETI, MINATEC Campus, F-38054 Grenoble, France \\ ${ }^{3}$ CEA, iRTSV-Biologie Grande Echelle, F-38054 Grenoble, France \\ ${ }^{4}$ INSERM, U1038, F-38054 Grenoble, France \\ ${ }^{*}$ The authors have equally contributed to this work. \\ fabien.momey@cea.franthony.berdeu@cea.fr
}

\begin{abstract}
New microscopes are needed to help realize the full potential of 3D organoid culture studies. In order to image large volume of 3D organoid cultures while preserving the ability to catch every single cell, we propose a new imaging platform based on lensfree microscopy. We have built a lensfree diffractive tomography setup performing multi-angle acquisitions of 3D organoid culture embedded in Matrigel ${ }^{\circledR}$ and developed a dedicated 3D holographic reconstruction algorithm based on the Fourier diffraction theorem. With this new imaging platform, we have been able to reconstruct a $3 \mathrm{D}$ volume as large as $21.5 \mathrm{~mm}^{3}$ of a $3 \mathrm{D}$ organoid culture of prostatic RWPE1 cells showing the ability of these cells to assemble in 3D intricate cellular network at the mesoscopic scale. Importantly, comparisons with 2D images show that it is possible to resolve single cells isolated from the main cellular structure with our lensfree diffractive tomography setup.
\end{abstract}

(C) 2016 Optical Society of America

OCIS codes: (090.1970) Diffractive optics; (090.1995) Digital holography; (100.3010) Image reconstruction techniques; (170.0110) Imaging systems; (180.6900) Three-dimensional microscopy.

\section{References and links}

1. N. Scherf, and J. Huisken, "The smart and gentle microscope," Nature Biotechnology, 33(8), 815-818 (2015).

2. S. Vinjimore Kesavan, F. Momey, O. Cioni, B. David-Watine, N. Dubrulle, S. Shorte, E. Sulpice, D. Freida, B. Chalmond, J. M. Dinten, X. Gidrol, and C. Allier, "High-throughput monitoring of major cell functions by means of lensfree video microscopy," Sci. Rep. 4, Article number: 5942 (2014).

3. I. Pushkarsky, Y. Liu, W. Weaver, T.-W. Su, O. Mudanyali, A. Ozcan, and D. Di Carlo, "Automated single-cell motility analysis on a chip using lensfree microscopy," Sci. Rep. 4, Article number: 4717 (2014).

4. G. Zheng, S. A. Lee, Y. Antebi, M. B. Elowitz, and C. Yang, "The ePetri dish, an on-chip cell imaging platform based on subpixel perspective sweeping microscopy (SPSM)," Proc. Natl. Acad. Sci. U.S.A. 108(18), 1688916894. (2011).

5. M. E. Dolega, C. Allier, S. V. Kesavan, S. Gerbaud, F. Kermarrec, P. Marcoux, J.-M. Dinten, X. Gidrol, and N. Picollet-D'hahan, "Label-free analysis of prostate acini-like 3D structures by lensfree imaging," Biosens. Bioelectron., 49, 176-183, (2013).

6. S. O. Isikman, W. Bishara, S. Mavandadi, F. W. Yu, S. Feng, R. Lau, and A. Ozcan, "Lens-free optical tomographic microscope with a large imaging volume on a chip," Proc. Natl. Acad. Sci. U.S.A. 108(18), 7296-7301. (2011).

7. S. O. Isikman, A. Greenbaum, W. Luo, A. Coskun, and A. Ozcan, "Giga-pixel lensfree holographic microscopy and tomography using color image sensors," PLOS ONE 7(9), e45044 (2012). 
8. E. Wolf, "Three-Dimensional structure determination of semi-transparent objects from holographic data," Opt. Commun., 1(14), 153-156 (1969).

9. Y. Sung, W. Choi, C. Fang-Yen, K. Badizadegan, R. R. Dasari, and M. S. Feld, "Optical diffraction tomography for high resolution live cell imaging," Opt. Express, 17(11), 266-277 (2009).

10. O. Haeberlé, K. Belkebir, H. Giovaninni, and A. Sentenac, "Tomographic diffractive microscopy: basics, techniques and perspectives," J. Mod. Opt., 57(9), 686-699 (2010).

11. Y. Cotte, F. Toy, P. Jourdain, N. Pavillon, D. Boss, P. Magistretti, P. Marquet, and C. Depeursinge, "Marker-free phase nanoscopy," Nature Photon., 7, 113-117 (2013).

12. A. Kak and M. Slaney, Principles of computerized tomographic imaging, (IEEE Press, 1988).

13. J. R. Fienup, "Phase retrieval algorithms: a comparison,” Appl. Opt., 21(15), 2758-2769 (1982).

\section{Introduction}

The study of in vitro cell populations remains a challenging task if one needs to gather large quantitative and systematic data over extended period of time while preserving the integrity of the living sample. As discussed in [1], there is a need for a new microscopy technique that must be label-free and non-phototoxic to be as "gentle" as possible with the sample, and "smart" enough to observe the sample exhaustively at a variety of scales both in space and time. Lensfree video microscopy is addressing these needs in the context of 2D cell culture [2-4].

As scientists better understand the benefit of growing organoids in 3D and routinely adopt 3D culture techniques, lensfree imaging must also be adapted to 3D cultures. Therefore, the new challenging task is to extend lensfree microscopy techniques to the acquisitions and $3 \mathrm{D}$ reconstructions of large organoids structures [5-7]. The adaptation of lensfree microscopy techniques to $3 \mathrm{D}$ organoid cultures imaging is the scope of the present paper.

We first describe an experimental bench dedicated to lensfree diffractive tomography of 3D biological samples. Next, we present a 3D reconstruction algorithm based on diffractive tomographic microscopy methods [8-11] but applied here to lensfree microscopy. And as a first proof of principle, we present the 3D reconstruction of a RWPE1 prostatic cell culture in 3D within an extra-cellular matrix (Matrigel ${ }^{\circledR}$ ). The reconstructed volume is as large as $21.5 \mathrm{~mm}^{3}$ which allows to observe simultaneously a very large cellular networks and isolated cells.

\section{Materials and methods}

\subsection{Cell lines and $3 D$ organoid culture}

RWPE1 (ATCC CRL-11609) cells were derived from non-neoplastic human prostate epithelial cells immortalized with human papillomavirus. Cells were maintained in KSFM (Life Technologies, Carlsbad, CA, ref. 17005-075) supplemented with $5 \mathrm{ng} / \mathrm{mL}$ epidermal growth factor (EGF) and $50 \mu \mathrm{g} / \mathrm{mL}$ bovine pituitary extract. The cells were maintained in culture until approximately $70 \%$ confluency. For passaging, cells were washed with Dulbeccos $\mathrm{Ca} 2+\mathrm{Mg}$-free PBS (D-PBS, Life Technologies, ref. 14190) and incubated with $1 \mathrm{~mL}$ trypsin-EDTA (Lonza, Basel, CH, ref. CC-5012, $0.25 \mathrm{mg} / \mathrm{mL}$ ) for approximately 7 minutes. The trypsin was neutralized with $2 m L$ trypsin neutralizing solution (Lonza, ref. CC-5002), and the cells were recovered by centrifugation and counted using a Scepter 2.0 Handheld Automated Cell Counter (Millipore, Billerica, MA, ref. PHCC 20060). The RWPE1 cells were cultured in 3D with KSFM (Life Technologies, ref. 17005-075) supplemented with $50 \mathrm{ng} / \mathrm{mL}$ EGF and 2\% fetal bovine serum (FBS). The 3D culture was grown in Matrigel ${ }^{\circledR}$ (BD Biosciences, San Jose, CA, ref. 356231) according to a no top-coat protocol. Briefly, Matrigel ${ }^{\circledR}$ was thawed overnight and poured into 4-well $\left(160 \mu L\right.$ of Matrigel $\left.{ }^{\circledR}\right)$ or 8-well Labtek ${ }^{\circledR}\left(90 \mu L\right.$ of Matrigel $\left.^{\circledR}\right)$ plates on ice. For polymerization, Matrigel ${ }^{\circledR}$ was incubated for 30 minutes at $37^{\circ} \mathrm{C}$. Cells were seeded in half the final volume (500 $\mu \mathrm{L}$ or $250 \mu \mathrm{L}$ of culture media in 4-well or 8-well Labtek ${ }^{\circledR}$ respectively) and allowed to adhere for approximately 45 minutes. Then culture media was slowly poured 
over the attached cells and was changed every other day. All cells were routinely cultured in a humidified atmosphere with $5 \% \mathrm{CO}_{2}$ at $37^{\circ} \mathrm{C}$.

\subsection{Experimental bench}

Unlike 2D lensfree imaging, where only one image is required for retrieving the 2D object, the reconstruction of a $3 \mathrm{D}$ object from lensfree acquisitions requires to multiply the viewing angles. For this purpose, we have developed an experimental bench, illustrated on Fig. 1. It is composed of a semi-coherent illumination source (LED CREE, $\lambda=520 \mathrm{~nm}-$ ref. XLamp MCE RGBW MCE4CT) and CMOS sensor (IDS - $29.4 \mathrm{~mm}^{2}, 3840 \times 2748$ monochromatic pixels, pixel pitch $1.67 \mu m$ - ref. UI-1942LE-M).

The object and the sensor are static, the object being placed on top of the sensor at a distance of 1 to $3 \mathrm{~mm}$. The source is rotated with a rotation stage (Newport - ref. M-URM80APP, controlled by a Newport universal motion controller - ref. ESP300) about the $\varphi$-axis (see Fig. 1 and 2) and thus the illumination direction is tilted relative to the sensor plane. This configuration is the most adapted to 3D organoid culture in standard containers such as Petri dish or well plates.

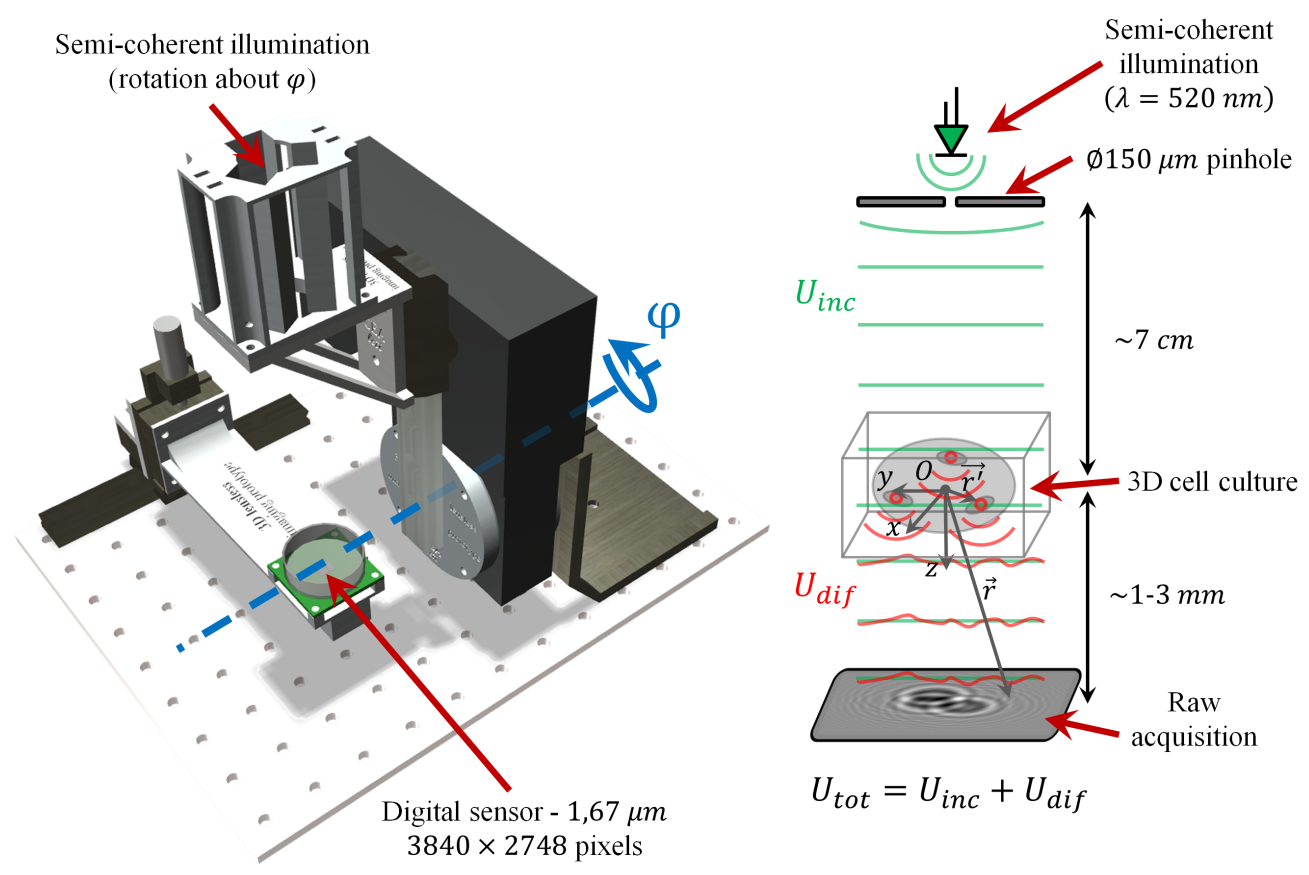

Fig. 1. Left-hand side - Experimental bench dedicated to lensfree diffractive tomography. Right-hand side - Optical schema of the system. The semi-coherent incident plane wave $U_{\text {inc }}$ passes through the sample volume. Each element of the volume diffracts the incident plane, behaving like secondary spherical sources, creating a diffracted wave $U_{d i f}$. The sensor records the intensity of their summation: $I_{\text {tot }}=\left|U_{\text {tot }}\right|^{2}=\left|U_{\text {inc }}+U_{\text {dif }}\right|^{2}$

\subsection{Reconstruction methods}

In the case of $3 \mathrm{D}$ lensfree imaging, the modelling of the wave $U_{\text {dif }}$ diffracted by the sample (see Fig. 1), defined in each point $\vec{r}=(x, y, z)$ of the space, is given by [8]: 


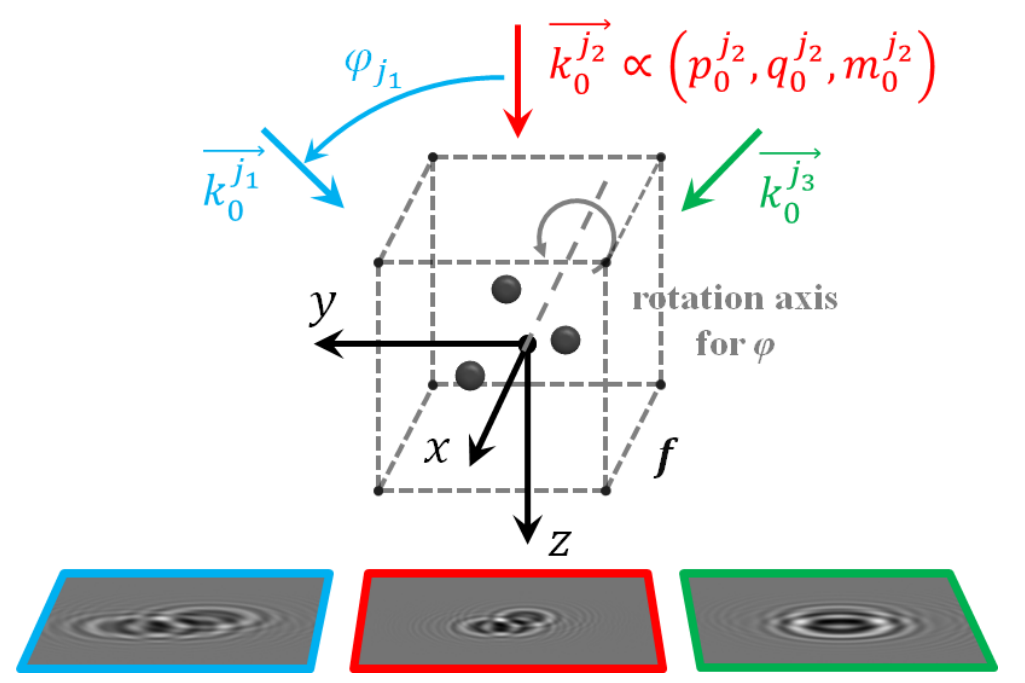

Fig. 2. Schema of three different acquisitions at the illumination angles $\varphi_{j_{1}}, \varphi_{j_{2}}$ and $\varphi_{j_{3}}$, with $\left(j_{1}, j_{2}, j_{3}\right) \in \llbracket 1, N \rrbracket^{3}$, where $N$ is the total number of acquisitions.

$$
U_{\text {dif }}(\vec{r})=\frac{-1}{4 \pi} \int_{\text {object }} f\left(\overrightarrow{r^{\prime}}\right) U_{\text {inc }}\left(\overrightarrow{r^{\prime}}\right) \frac{\exp \left(i k_{0}\left\|\vec{r}-\vec{r}^{\prime}\right\|\right)}{\left\|\vec{r}-\overrightarrow{r^{\prime}}\right\|} d^{3} \vec{r}^{\prime}=-\frac{1}{4 \pi}\left(f . U_{\text {inc }}\right) * h
$$

where $k_{0}=\frac{2 \pi n_{0}}{\lambda_{0}}$ is the wave number in an ambient medium of refractive index $n_{0}$ and $h(\vec{r})=\frac{1}{\|\vec{r}\|} \exp \left(i k_{0}\|\vec{r}\|\right)$ is the convolution kernel characteristic of spherical wave propagation. The scattering potential $f(\vec{r})$ is the 3D object to be reconstructed. It is related to the complex refractive index of the object $n(\vec{r})$ by the formula:

$$
f(\vec{r})=-k_{0}^{2}\left(\left(\frac{n(\vec{r})}{n_{0}}\right)^{2}-1\right)
$$

These equations are valid when the overall phase delay introduced by the sample is weak (see [9]). In the case of 3D cell culture, this requirement is generally not met since these samples feature objects with dimensions larger than $l=20 \mu \mathrm{m}$. In water, for a typical cell refractive index of 1.35, the difference of refractive index is $\delta n=0.02$, introducing a phase delay of $2 \pi l \delta n / \lambda$. In visible light $(\lambda \simeq 550 \mathrm{~nm})$, this phase delay is greater than $\pi$ and therefore cannot be supposed to be weak. In the present work, we decided to use these equations nonetheless to determine if some information, interesting on the biological point of view such as positions, dimensions and shapes of the objects could be estimated. As a drawback of the methodology, it is clear that quantitative results such as refractive indexes or optical lengths cannot be obtained.

\subsubsection{Algorithm}

Our reconstruction algorithm recovers $f$ based on $N$ multiple acquisitions at different angles $\left(\varphi_{j}\right)_{j \in \llbracket 1, N \rrbracket}$ (see Fig. 2), and is based on the Fourier diffraction theorem, widely used in the field of diffractive tomographic microscopy [9-11]. This latter links $\hat{U}_{d i f}$, the 2D Fourier transform of $U_{d i f}$ to $\hat{f}$, the 3D Fourier transform of the object $f$. At a given illumination angle $\varphi_{j}$ on the sensor plane $z=z_{s}$, Eq. (1) becomes (see $[8,12]$ ): 


$$
\hat{f}\left(\alpha^{j}, \beta^{j}, \gamma^{j}\right)=\underbrace{4 i \pi w \cdot \exp \left(-2 i \pi w z_{s}\right)}_{H_{z s}^{\left\{\vec{k}_{0}^{j} ; \lambda_{0}\right\}}} \hat{U}_{d i f}^{j}\left(u, v ; z_{s}\right)
$$

with, $\left\{\begin{array}{l}\alpha^{j}=u-\frac{n_{0} \cdot p_{0}^{j}}{\lambda_{0}} \\ \beta^{j}=v-\frac{n_{0} \cdot q_{0}^{j}}{\lambda_{0}} \\ \gamma^{j}=w-\frac{n_{0} \cdot m_{0}^{j}}{\lambda_{0}}\end{array}\right.$ and $w=\sqrt{\frac{n_{0}^{2}}{\lambda_{0}^{2}}-u^{2}-v^{2}}$, where $(u, v)$ and $(\alpha, \beta, \gamma)$ correspond re-

spectively to the Fourier space coordinates of the sensor and the object. $\left(p_{0}^{j}, q_{0}^{j}, m_{0}^{j}\right)$ is the orientation of the $\varphi_{j}$ illumination, i.e. the orientation of the wave vector $\vec{k}_{0}{ }^{j}$ :

$$
\left\{\begin{array}{l}
p_{0}^{j}=0 \\
q_{0}^{j}=\sin \varphi_{j} \\
m_{0}^{j}=\cos \varphi_{j}
\end{array}\right.
$$

Notice that the Fourier transform is defined for a given function $g$ as:

$$
\hat{g}(u)=\int_{-\infty}^{\infty} g(x) \exp (-2 i \pi u x) d x
$$

Figure 3 illustrates the Fourier diffraction theorem: a geometrical description is that the 2D Fourier transform $\hat{U}_{d i f}^{j}$ of a given diffracted wave $U_{d i f}^{j}$ is mapped on a spherical cap (2D surface, also called Ewald's sphere) depending on $\left(\vec{k}_{0}^{j} ; \lambda_{0}\right)$ inside the 3D frequency space of the object of interest $f$. To actually map the uniform 3D discrete Fourier space of $\hat{f}$, the discrete Fourier transform of each projection is computed. The position of each calculated 2D Fourier coefficient on its spherical cap is then determined and its value is associated to the nearest neighbour into this uniform frequency volume. Once all the 2D Fourier transforms of projections are mapped, it results to gaps within the 3D frequency space, as it can be seen in the medallion of Fig. 3. In this paper, it was decided not to apply any interpolation method to generate a fully filled 3D frequency support. Finally a 3D inverse discrete Fourier transform retrieves the object $f$.

In sum, the algorithm operates as follows:

$$
f=\mathscr{F}_{3 D}^{-1}\left[\left.S\left(H_{z_{s}}^{j} \cdot \mathscr{F}_{2 D}\left[M_{0}^{j} \cdot U_{d i f}^{j}\right]\right)\right|_{\left\{\vec{k}_{0}^{j} \mid j=1 \rightarrow N\right\}}\right]
$$

where $f$ constitutes the 3D image of size $N_{x} \times N_{y} \times N_{z}$ to be reconstructed. $\mathscr{F}_{3 D}^{-1}$ and $\mathscr{F}_{2 D}$ are respectively the inverse 3D and direct $2 \mathrm{D}$ discrete Fourier transforms. They are performed by the FFT algorithm. $S$ stands for the mapper into the 3D frequency space of the $N_{j}$ spherical caps corresponding to $N_{j}$ projections $U_{d i f}^{j}$ to which a given illumination wave vector $\vec{k}_{0}^{j}$ is associated. The projections are 2D complex images of size $N_{d_{x}} \times N_{d_{y}}$ corresponding to the sensor's size, with a pixel pitch denoted $\Delta_{d}$, which is the same in each direction (see Fig. 1). $M_{0}^{j}$ is the modulation of $U_{d i f}^{j}$ in the spatial domain corresponding to the translation of the spherical cap from the sensor's coordinate space $(u, v)$ to the object's coordinate space $\left(\alpha^{j}, \beta^{j}, \gamma^{j}\right)$ in the frequency domain in Eq. (3) (see Fig. 3):

$$
M_{0}^{j}(x, y)=\exp \left(-2 i \pi\left(\frac{n_{0} \cdot p_{0}^{j}}{\lambda_{0}} x+\frac{n_{0} \cdot q_{0}^{j}}{\lambda_{0}} y\right)\right)
$$




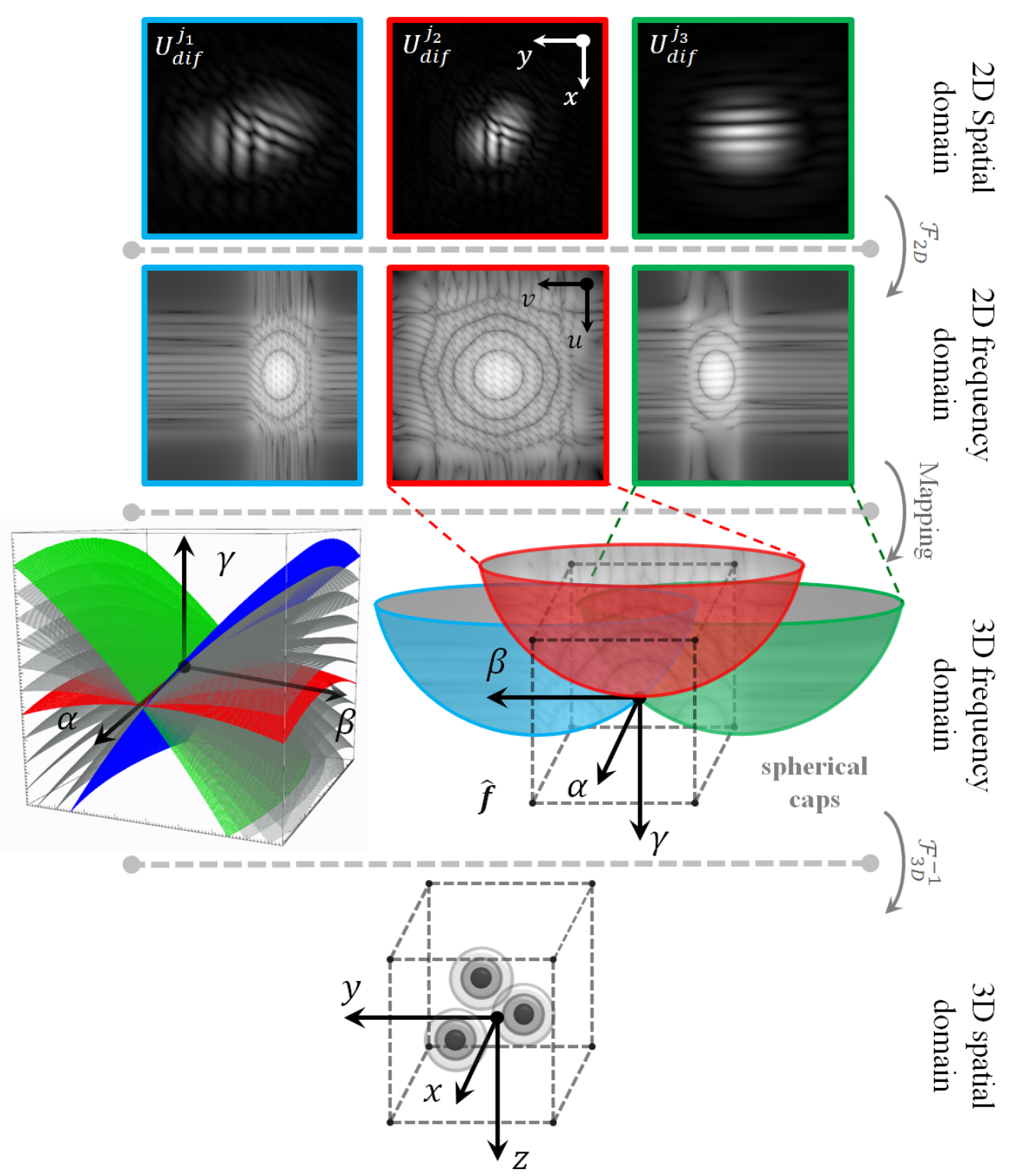

Fig. 3. Illustration of the Fourier diffraction theorem with the notations of Fig. 2. The 3D frequency space of the object of interest $f$ is mapped with the 2D Fourier transform of the projections $U_{d i f}^{j_{1}}, U_{d i f}^{j_{2}}$ and $U_{d i f}^{j_{3}}$. An example of a Fourier region which is actually filled by the algorithm is given in the medallion in the $3 \mathrm{D}$ frequency domain part of the figure with the parameters used in section 3 to reconstruct a given region of interest: a volume of $1.34 \times 1.34 \times 1.34 \mathrm{~mm}^{3}$ with a voxel size of $1.67 \times 1.67 \times 3.34 \mu \mathrm{m}^{3}$ at $\lambda=520 \mathrm{~nm}$ with an illumination angle varying from $-30^{\circ}$ (blue) to $30^{\circ}$ (green) with a step angle of $5^{\circ}$. The red cap corresponds to the region map with the normal illumination.

2.3.2. An approximation for $\left(U_{d i f}^{j}\right)_{j \in \llbracket 1, N \rrbracket}$

In our case, only the intensity of the total transmitted wave $I_{t o t}^{j}=\left|U_{t o t}^{j}\right|^{2}$ is acquired by the sensor, where $U_{t o t}=U_{i n c}+U_{d i f}$ (see Fig. 1 and 2). Hence we have to approximate the diffracted 
wave $U_{d i f}^{j}$ from $I_{\text {tot }}^{j}$. To do so, we first normalize $I_{\text {tot }}^{j}$ so that the background value, corresponding to the unperturbed incident wave, is equal to 1 . We note this normalized projection $\bar{I}_{\text {tot }}^{j}$. We multiply the square root of $\bar{I}_{t o t}^{j}$ by a phase ramp $\exp \left(\overrightarrow{i k_{0}^{j}} \cdot \vec{r}\right)$ to take into account the non-uniform phase shift induced by the tilted incidence of $\overrightarrow{k_{0}^{j}}$ relative to the sensor plane. Then we subtract the theoretical incident plane wave $U_{i n c}^{j}=\exp \left(\overrightarrow{i k_{0}^{j}} \cdot \vec{r}\right)$ :

$$
U_{d i f}^{j} \simeq \sqrt{\bar{I}_{t o t}^{j}} \exp \left(\overrightarrow{i k_{0}^{j}} \cdot \vec{r}\right)-U_{i n c}^{j}
$$

This formula is schematically represented on Fig. 4. This is a needed step in the presented methods. Indeed, the phase is very important since, inter alia, it contains the inclination of the wavefront. Without this information, the mapping of the Fourier space with Eq. (5) cannot work because the computed 2D spectra will be shift in the Fourier space. Equation (7) allows to reintroduce the information of the illumination angle in the data even if the simulated phase is just an approximation of the real one.

This calculation does not totally compensate the lack of phase information at the sensor plane. In particular, the phase distortion introduced by the object is not taken into account and could be approximated by means of a phase retrieval algorithm in order to diminish the artefactual twin image [13].

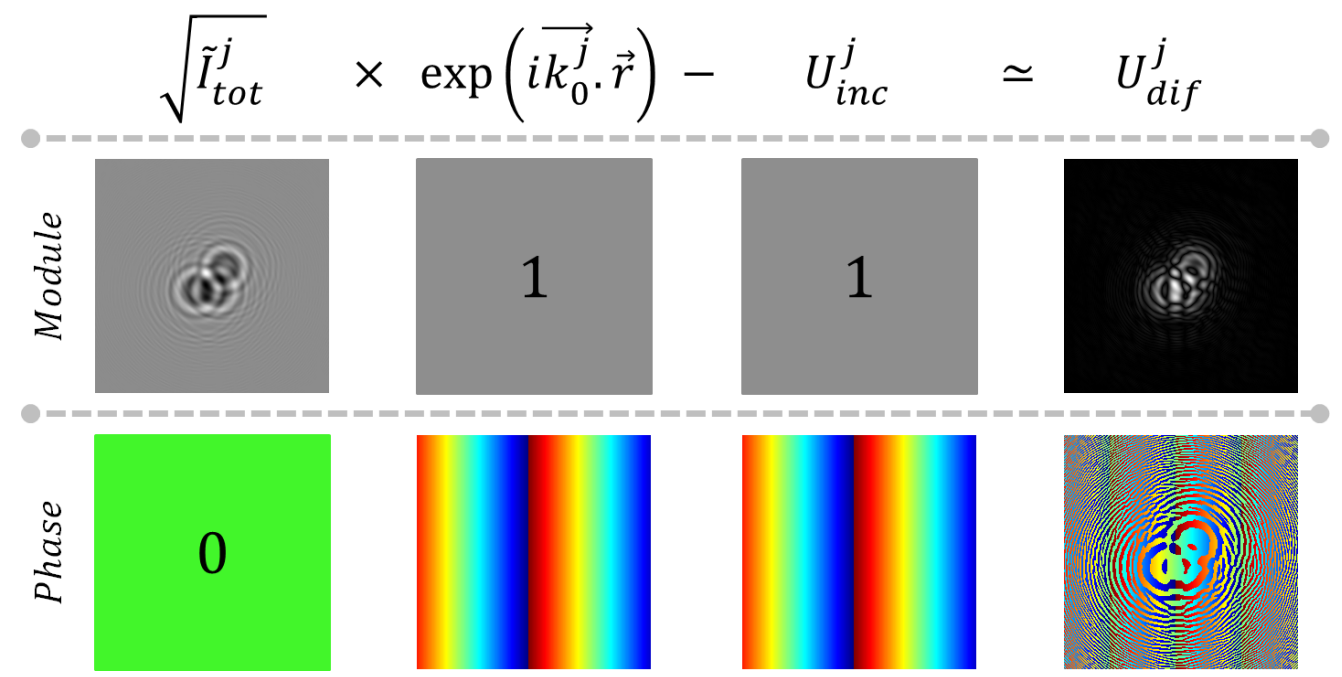

Fig. 4. Illustration of the steps to get an approximation of $U_{d i f}^{j}$ for a given $j$.

\subsubsection{Reconstruction of regions of interest}

If we take $1.67 \times 1.67 \times 1.67 \mu \mathrm{m}^{3}$ voxels based on the pixel pitch of the sensor (see Fig. 1), the size of the 3D reconstructed image is very huge - up to several tens of gigabytes - if we want to maintain the same resolution in each direction $\left(N_{x} \approx N_{d_{x}}, N_{y} \approx N_{d_{y}}\right.$ and $N_{z} \approx N_{d_{x}}$ or $N_{d_{y}}$ ). This could be too expensive for conventional computers and could cause memory overflows. To address this problem, we can extract from the data a region of interest (ROI) and reconstruct it separately. The extraction process of a ROI is illustrated on Fig. 5. The ROI is defined on the projection corresponding to $\varphi=0^{\circ}$, i.e. where the incidence of illumination is normal to the sensor. Knowing the altitude $z_{s}$ and the angle $\varphi^{j}$, we can predict where the particular structures 
in the ROI have been shifted in the other projections, due to the tilted incidence. The shift is theoretically known and equals:

$$
\left\{\begin{aligned}
x_{0}^{j} & =0 \\
y_{0}^{j} & =z_{s} \tan \varphi^{j}
\end{aligned}\right.
$$

The centering of the ROI whatever the angle of view implies to modify the algorithm to indicate the absolute position of the ROI on each projection. As it is a translation in the spatial domain, this yields to a modulation of the Fourier transform of the projection as a function of $\left(x_{0}^{j}, y_{0}^{j}\right)$, giving from Eq. (5):

$$
f=\mathscr{F}_{3 D}^{-1}\left[\left.S\left(H_{z s}^{j} \cdot D_{0}^{j} \cdot \mathscr{F}_{2 D}\left[M_{0}^{j} \cdot U_{d i f_{R O I}}^{j}\right]\right)\right|_{\left\{\vec{k}_{0}^{j}, x_{0}^{j}, y_{0}^{j} \mid j=1 \rightarrow N_{j}\right\}}\right]
$$

where

$$
D_{0}^{j}(u, v)=\exp \left(-2 i \pi\left(u x_{0}^{j}+v y_{0}^{j}\right)\right)
$$

and

$$
M_{0}^{j}(x, y)=\exp \left(-2 i \pi\left(\frac{n_{0} \cdot p_{0}^{j}}{\lambda_{0}}\left(x+x_{0}^{j}\right)+\frac{n_{0} \cdot q_{0}^{j}}{\lambda_{0}}\left(y+y_{0}^{j}\right)\right)\right) .
$$

As a first approximation, we consider that the emitted light propagates in a homogeneous medium. As a result, it follows a straight path until it is diffracted by the objects of interest. However, our setup induces several refractive indexes' changes (air, culture medium, glass) along the light path. This causes uncertainties on the effective shift $\left(x_{0}^{j}, y_{0}^{j}\right)$ as a function of the illumination angle $\varphi^{j}$. Moreover, the altitude $z_{s}$ is not known absolutely and has to be estimated with a focusing algorithm using Fresnel backpropagation at incidence $\varphi=0^{\circ}$. As a solution, we use a registration algorithm to correctly center the extracted ROIs so that the projections match the theoretical shift. We only consider rigid translations, ignoring the fact that the holograms are also stretched differently as a function of the light incidence.

\section{Results}

We have acquired tomographic data of a culture of prostatic cells RWPE1 in Matrigel ${ }^{\circledR}$, over 61 angles from $-30^{\circ}$ to $30^{\circ}$ with an angular pitch of $1^{\circ}$. Figure 5 shows 3 projections at $\varphi=-30^{\circ}$, $\varphi=0^{\circ}$ and $\varphi=30^{\circ}$. We have reconstructed a large volume of $4 \times 4 \times 1.34 \mathrm{~mm}^{3}$ showing that the cells interestingly tend to form a structured network.

To reconstruct such a large volume, we worked by pieces of $1.34 \times 1.34 \times 1.34 \mathrm{~mm}^{3}$, i.e. nine reconstructions. Thus we have divided the data into nine adjacent ROIs of size $1.34 \times$ $1.34 \mathrm{~mm}^{2}(800 \times 800$ pixels), as illustrated on Fig. 6 . The registration algorithm for the centering of each projection was applied relative to the central ROI (dash red on Fig. 6), as well as the focusing algorithm to estimate $z_{s}$, the distance between the sensor plane and the cell culture. The eight other ROIs remain adjacent to this one whatever the angle of view, to maintain the continuity in the 3D reconstruction. Each reconstructed 3D image had a size of $800 \times 800 \times 400$ voxels with the sampling rate $1.67 \times 1.67 \times 3.34 \mu \mathrm{m}^{3}$. Owing this decoupling task, a single ROI reconstruction has a size of two gigabytes in double precision which can be handled on a desktop computer. As a result the global 3D image has a size of $2400 \times 2400 \times 400$ voxels with the same sampling rate, this whole image having a size close to 20 gigabytes.

The whole reconstructed volume $f$ is shown on Fig. 7. As it is a complex image, we only visualize its imaginary part which gave us the most observable information. Three different 


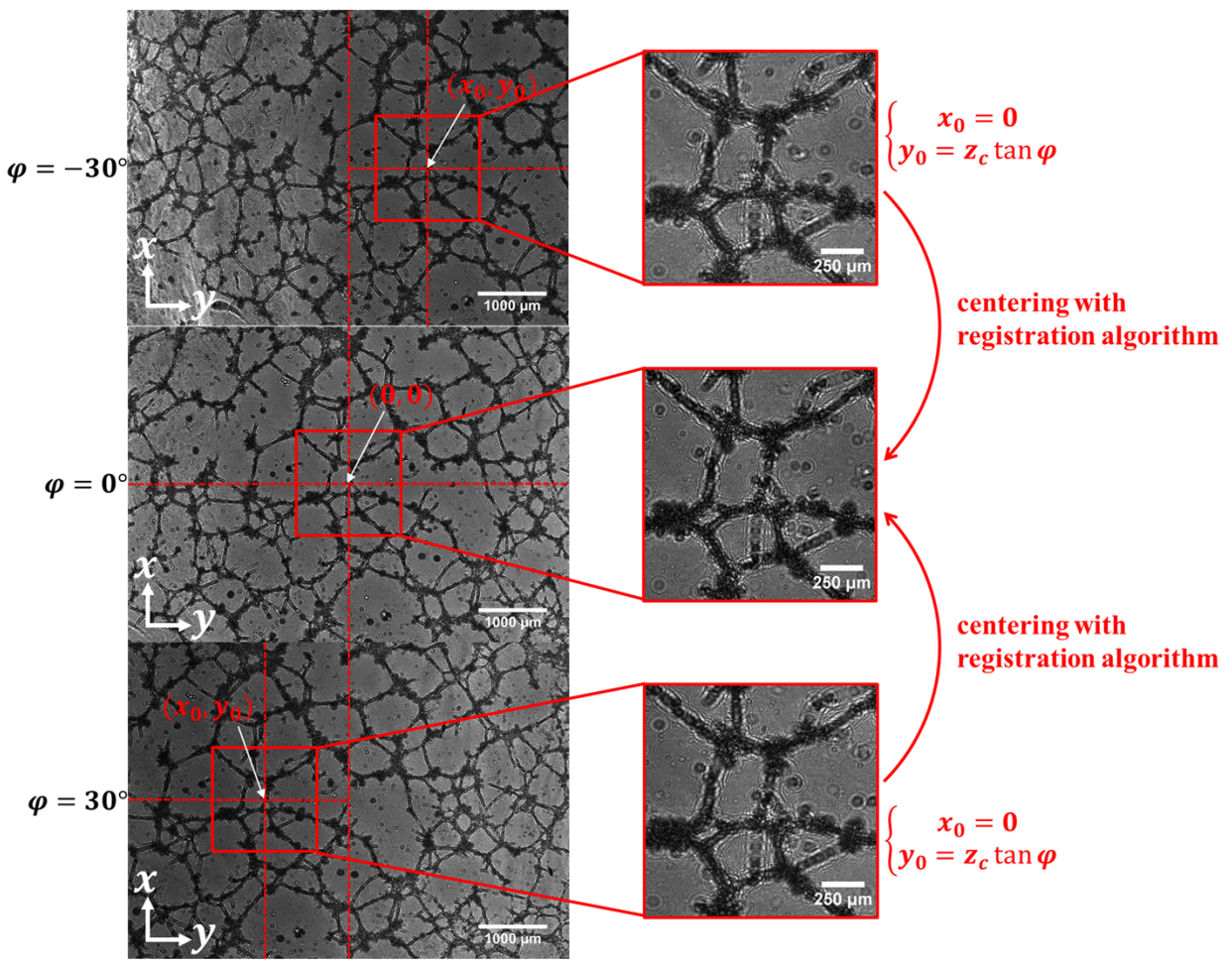

Fig. 5. 3D lensfree data of a culture of prostatic cells RWPE1 in Matrigel ${ }^{\circledR} .61$ angles of view were acquired from $-30^{\circ}$ to $30^{\circ}$ with an angular pitch of $1^{\circ}$ around the $x$ axis. The illumination wavelength is $520 \mathrm{~nm}$. A region of interest (ROI) is extracted on each projection and centered by registration. This aims at performing a localized reconstruction to save computation from RAM overflows. The centering has to be taken into account in the algorithm (Eq. (5)).

sectional views - $x y$ (center), $x z$ (right) and $y z$ (bottom) - and 3D views (see Visualizations 1 and 2) show that the network formed by the cells is well focused at precise altitudes in the volume. The network fits a surface which is not horizontal, which demonstrates that the reconstruction algorithm effectively operates in 3D: isolated objects are also focused apart from the network at independent altitudes. Some of them are pointed out by red arrows on Fig. 7.

The central ROI and the ROI in the top-right corner of the reconstructed volume shown on Fig. 7 (equivalently the central and bottom-right ROIs on the raw data on Fig. 6) and are further detailed on Fig. 8 and 9. Each of these figures represents the three sectional views - $x y$ (center), $x z$ (right) and $y z$ (bottom) - and a 3D view of the ROI and two specific patterns such as a cell or an organoid which are pointed by arrows in the ROIs. The rings around the focused objects on the $x y$ views are artefactual: this is the well-known artefactual phenomenon of twin image, induced by the lack of absolute phase information in the data.

To analyse the performances of our setup and 3D reconstruction algorithm to recover unambiguously biological objects, a comparative study with $2 \mathrm{D}$ reconstructions from $2 \mathrm{D}$ lensfree data acquired at the normal illumination $\left(\varphi=0^{\circ}\right)$ was conducted. The algorithm used for performing the 2D reconstructions has been presented in [2], and has already shown, as a $2 \mathrm{D}$ lensfree imaging application, its ability to recover single cells and organoids while removing the twin images $[2,5]$. 


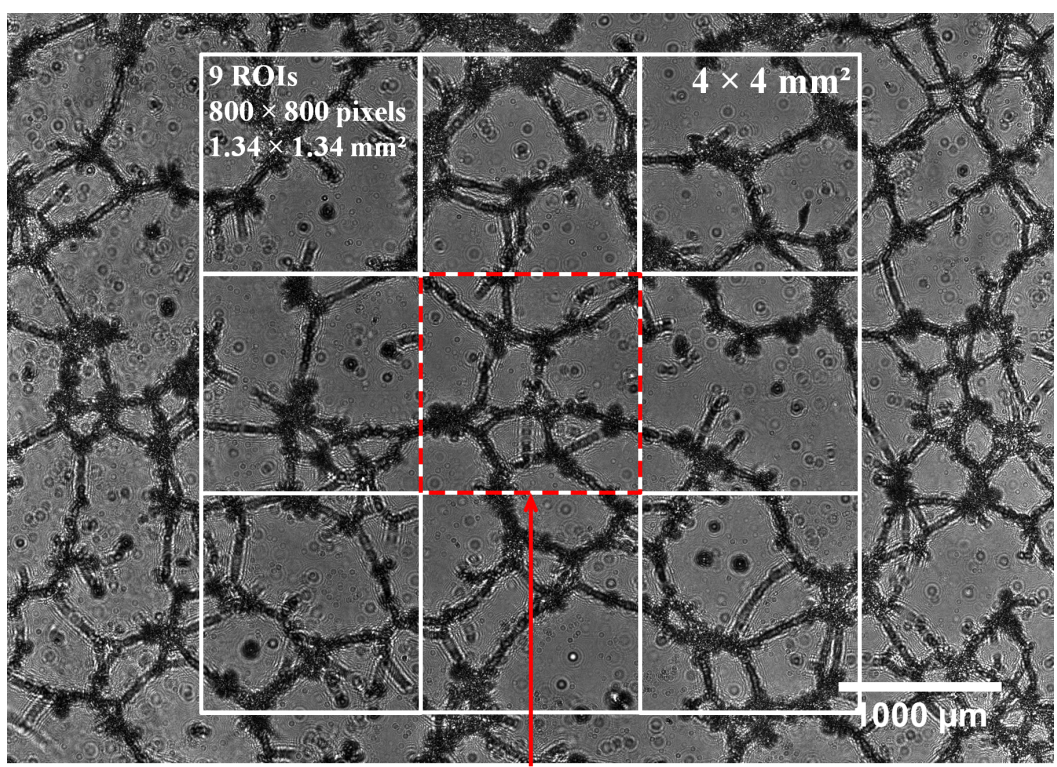

registration and focus reference

Fig. 6. Extraction of 9 ROIs of size $1.34 \times 1.34 \mathrm{~mm}^{2}(800 \times 800$ pixels $)$ for the piecewise $3 \mathrm{D}$ reconstruction of the 3D culture of prostatic cells RWPE1 presented on Fig. 5.

Figure 10 shows the 2D reconstruction of a region of the studied field of view. We can observe the efficiency of the reconstruction algorithm to recover the biological scene showing branching networks, single organoids and cells. To perform the comparisons with the 3D reconstruction, three objects were selected from the objects highlighted Fig. 8 and 9: two cells (see ROI 1 and ROI 2 on Fig. 10) and one organoid (see ROI 3 on Fig. 10). Two axial cut profiles respectively in $x$ and $y$ directions were taken for both the 2D and 3D reconstructions, and one cut profile in the $z$ direction for the 3D reconstruction. These cut profiles are illustrated on the three graphs on Fig. 10. As a first observation, we can see that axial profiles are globally equivalent in 2D and 3D, allowing to measure approximatively cell sizes of $\sim 15 \mu \mathrm{m}$ for ROI 2 and $\sim 30 \mu \mathrm{m}$ for ROI 1 considering the width of the central lobe of the profiles (the secondary lobes are known to be twin artefacts), which is consistent with typical cell sizes. For the organoid, the profiles are more erratic but we can distinguish a kind of a plateau curve, the width of which is measured at $\sim 90 \mu \mathrm{m}$. As expected, the observations are different in the $z$ direction and the resolution is clearly degraded: whereas the objects must have a global isotropic behaviour in terms of sizes in 3D, our measures give $\sim 80 \mu \mathrm{m}$ for the cell in ROI $1, \sim 115 \mu \mathrm{m}$ for the cell in ROI 2 and $\sim 200 \mu \mathrm{m}$ for the organoid in ROI 3. Moreover, the disparities of the measures for the cells tend to show that the apparent resolution highly depend on the position of the object in the field of view: the more we go away from the centre (from ROI 1 to ROI 2), the worse is the resolution in the $z$ direction. Note that the term of "resolution" that we employ has not to be confused with the strict definition of optical resolution. These are empirical measures that allow us to conclude that single biological objects are effectively and unambiguously identified in the $3 \mathrm{D}$ reconstruction.

Uncertainties in the calibration of the setup and the projection model can explain some artefacts in the image and the previous quantifications of Fig. 10. For example we did not take into account the stretching of holograms in the registration of the projections which depends of the lighting direction. Thus some misalignment can be present after the registration step. Moreover 
(a)
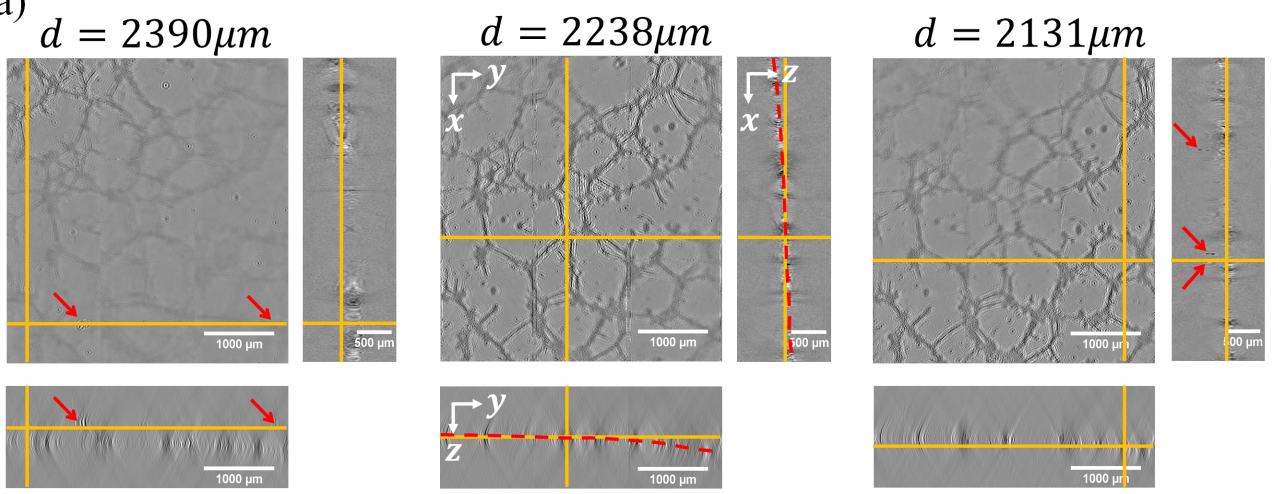

(b)
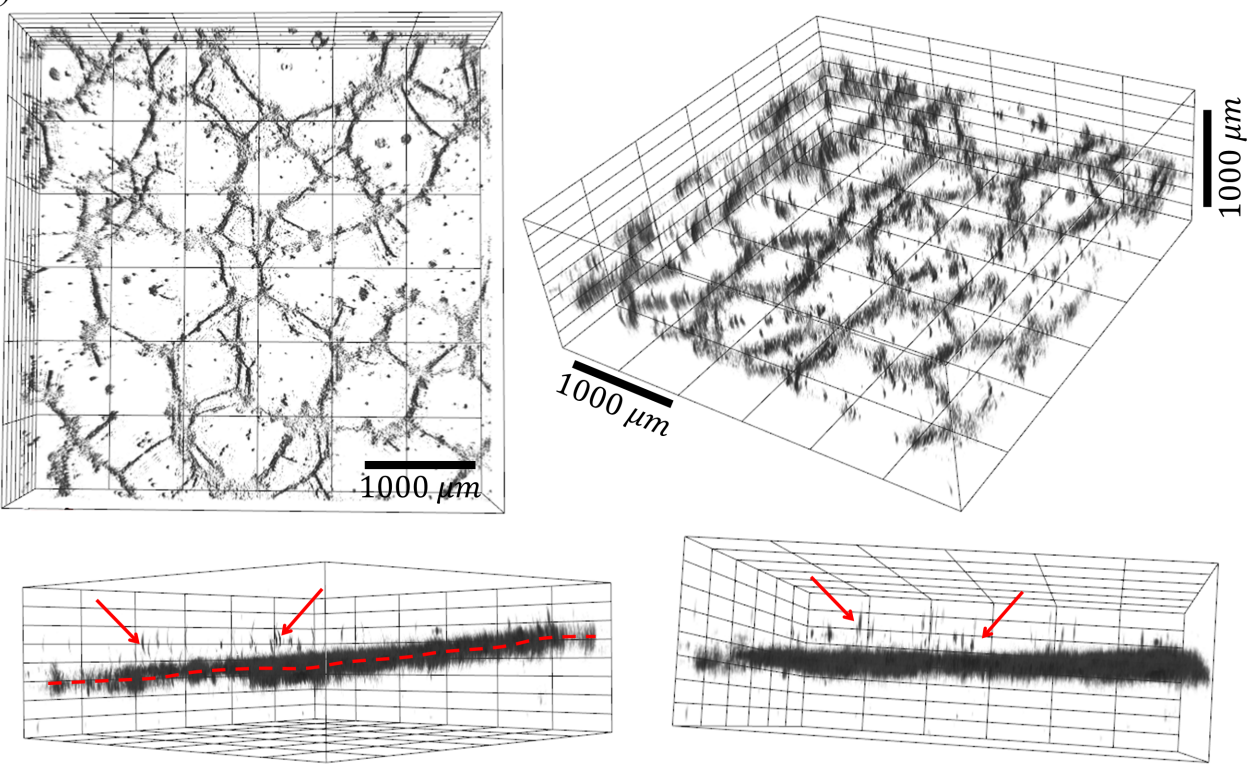

Fig. 7. Piecewise 3D reconstruction (imaginary part of $f)$ of a large volume $(4 \times 4 \times$ $1.34 \mathrm{~mm}^{3}$ ) of the culture of prostatic cells RWPE1 from tomographic data presented on Fig. 5. (a) 3 sectional views - $x y$ (center), $x z$ (right) and $y z$ (bottom) - are shown, each one focused at a given distance $d=z_{s}-z$ from the sensor. (b) 3D views of the reconstruction from different observation points (see Visualizations 1 and 2). The dash red curves indicate the tilt of the network relative to the horizontal plane. The red arrows point out isolated objects that are not focused at the same altitude as the network.

uncertainties remain on these lighting directions, due to the multiple refractions in between the different propagating media (air, culture medium, sensor glass, etc.) which change the illumination orientations. The induced artefacts then get worse as the distance from the center increases since the mistakes on the objects positioning grow with this distance. That is why the artefactual bow shape around the single objects get worse on the side, as one can see comparing Fig. 8 and 9 and the profiles on Fig. 10.

These critical points highlighted by our demonstrative results will be addressed in future works to allow us a precise quantification of the performances of 3D lensfree imaging, e.g. the effective optical resolution in all directions. 
(a)
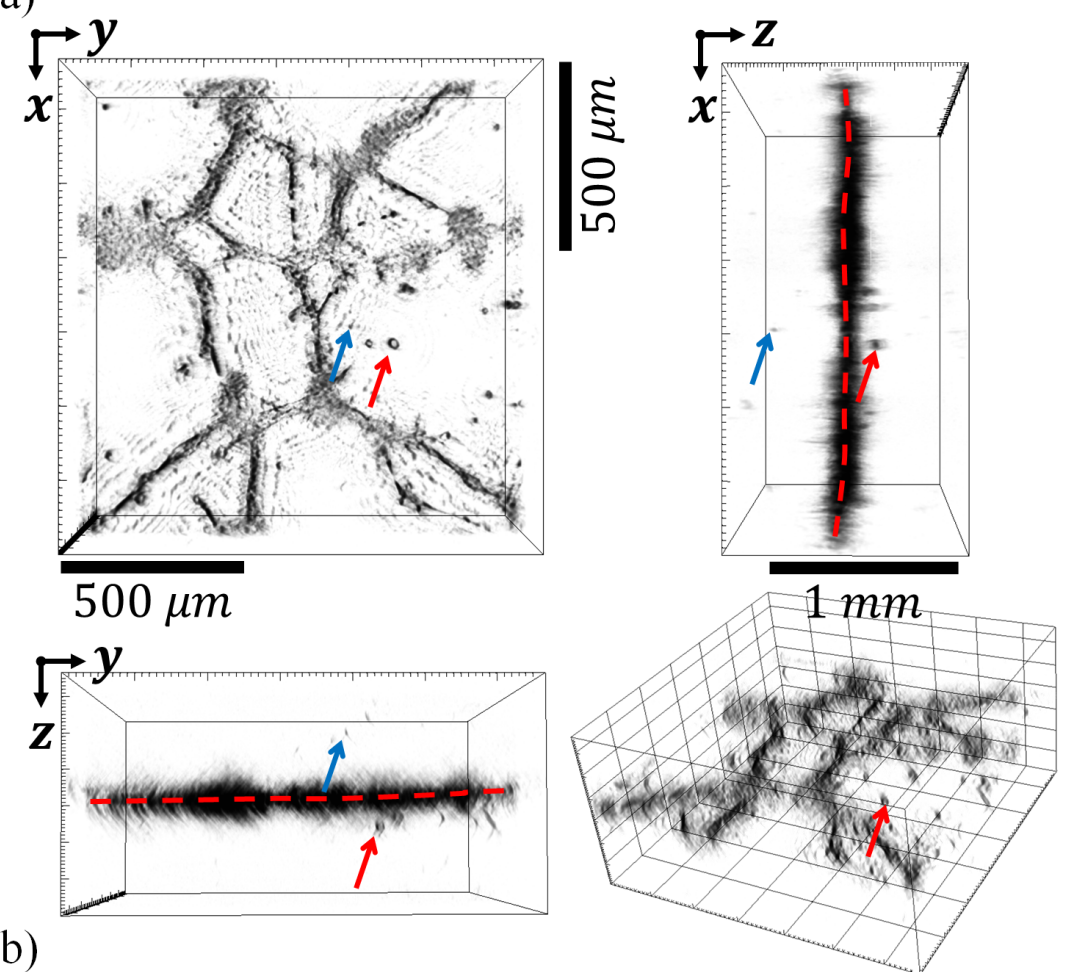

(b)
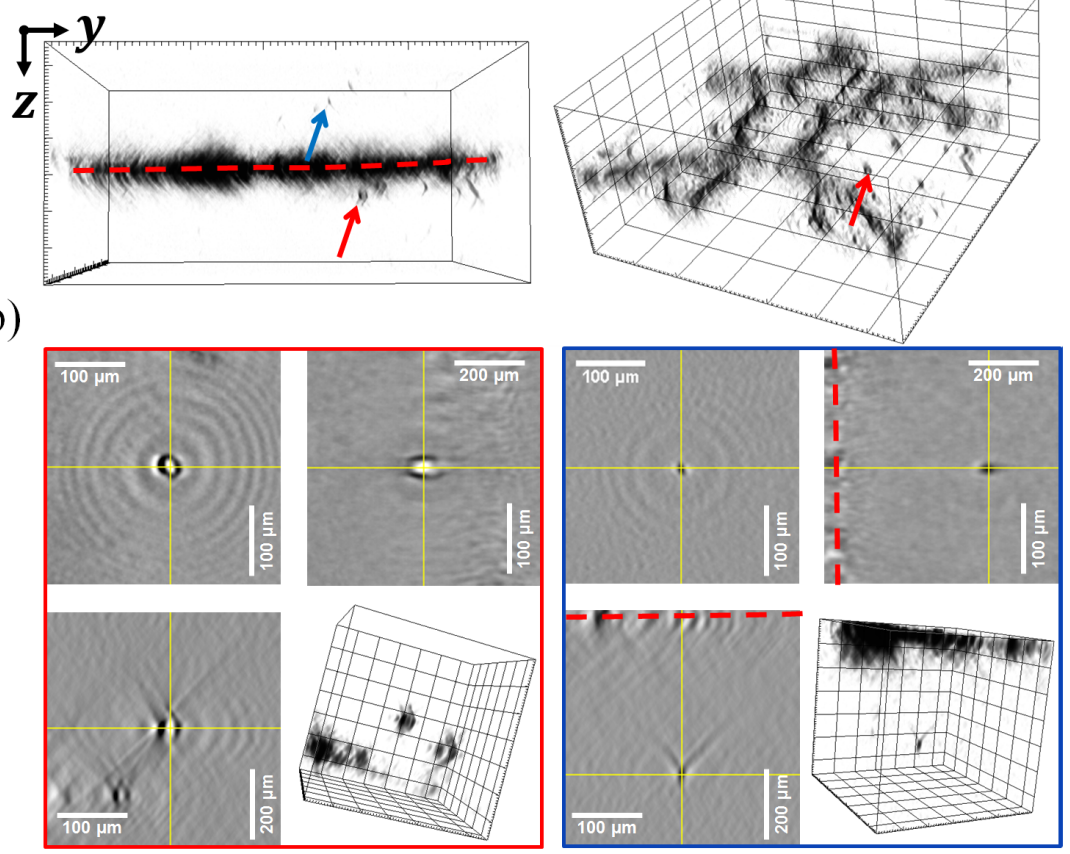

Fig. 8. ROI at the center of the piecewise $3 \mathrm{D}$ reconstruction of the volume of Fig. 7. (a) 3 sectional views - $x y$ (center), $x z$ (right) and $y z$ (bottom) - and a 3D view of the ROI. (a) same representation rules on two specific organoids at the full resolution of the reconstruction (see Visualizations 3, 4 and 5). These are pointed by arrows on the global view. The dashedline indicates the network plane. The red arrow points toward a $33 \times 38 \times 42 \mu \mathrm{m}^{3}$ object and the blue arrow toward a $16 \times 17 \times 40 \mu \mathrm{m}^{3}$ object.

\section{Conclusion}

In this paper, we have presented a novel technique for the imaging of 3D organoid cultures: lensfree diffractive tomography. Based on lensfree imaging and digital holography principles, we have demonstrated that it is possible to reconstruct large volumes up to several $\mathrm{mm}^{3}$, by multiplying the angles of view around the object of interest. We have developed a reconstruction 
(a)
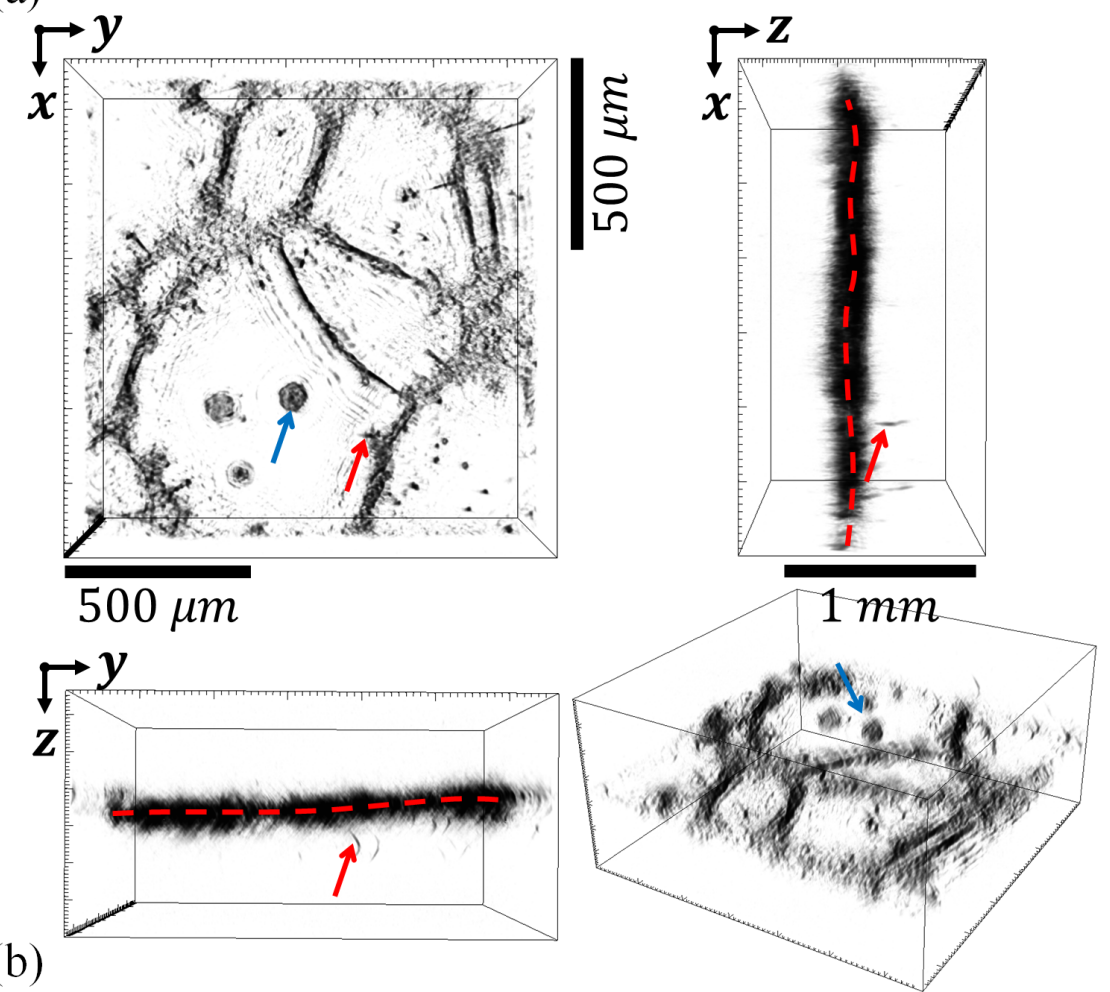

(b)
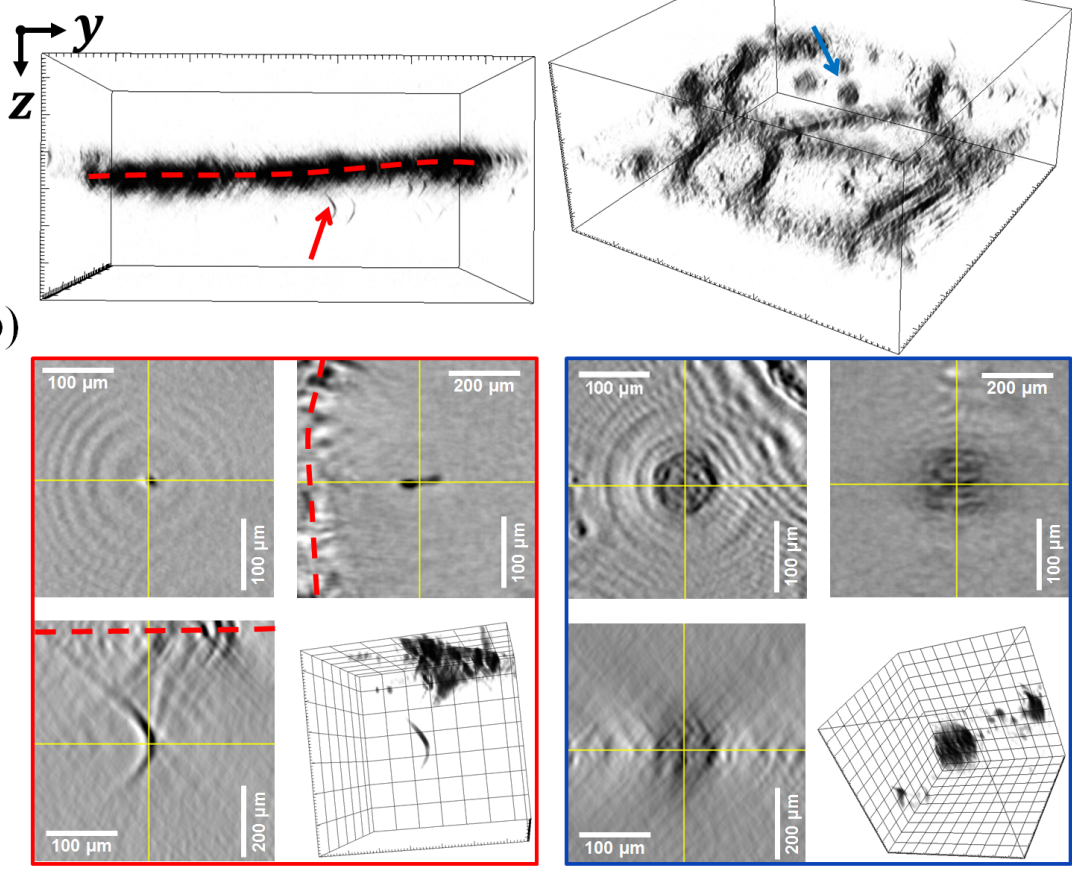

Fig. 9. ROI at the to right corner of the piecewise 3D reconstruction of the volume of Fig. 7. (a) 3 sectional views - $x y$ (center), $x z$ (right) and $y z$ (bottom) - and a 3D view of the ROI. (b) same representation rules on two specific organoids at the full resolution of the reconstruction (see Visualizations 6, 7 and 8). These are pointed by arrows on the global view. The dashed-line indicates the network plane. The red arrow points toward a $12 \times 43 \times$ $96 \mu \mathrm{m}^{3}$ object and the blue arrow toward a $80 \times 80 \times 100 \mu \mathrm{m}^{3}$ object.

algorithm based on the direct inversion of the data in the frequency domain using the known Fourier diffraction theorem. We have reconstructed a large volume $\left(4 \times 4 \times 1.34 \mathrm{~mm}^{3}\right)$ of a culture of RWPE1 prostatic cells in Matrigel ${ }^{\circledR}$. As a result we have been able to observe focused objects of a few tens of microns (cells and network branches).

These first results tend to demonstrate that it is possible to acquire knowledge on the posi- 

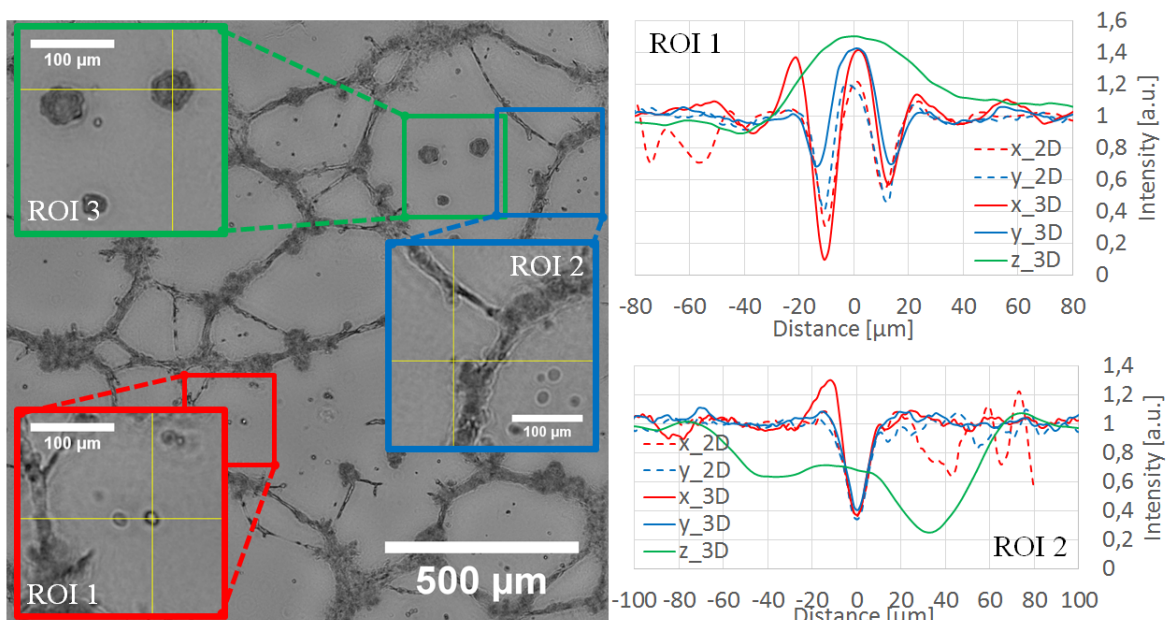

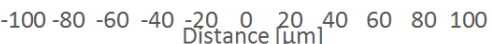

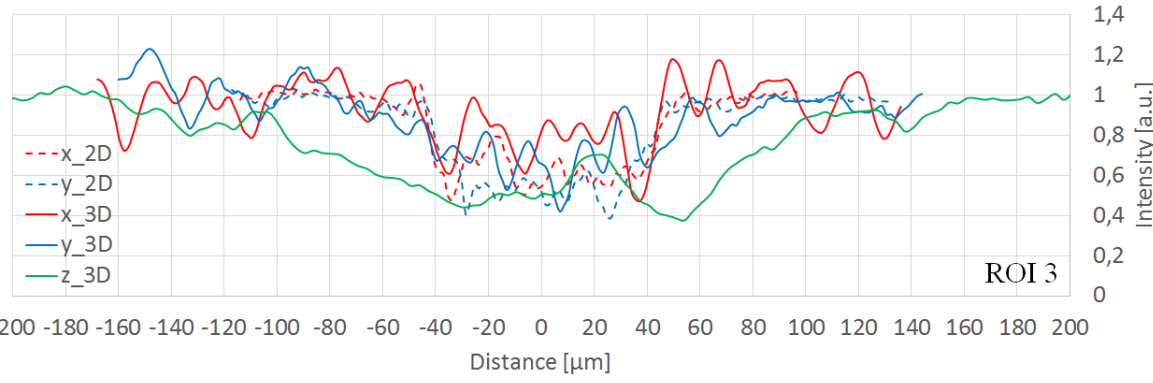

Fig. 10. 2D reconstruction from $2 \mathrm{D}$ lensfree data acquired on the $3 \mathrm{D}$ culture of prostatic cells RWPE1 presented on Fig. 5. Comparisons of two axial cut profiles respectively in $x$ and $y$ directions taken on both 2D and 3D reconstructions, and one cut profile in the $z$ direction taken on the 3D reconstruction, for three biological objects: two cells, one identified on Fig. 8 (ROI 1 in red) and one identified on Fig. 9 (ROI 2 in blue) and one organoid identified on Fig. 9 (ROI 3 in green). The dashed and solid curves correspond respectively to the $2 \mathrm{D}$ and $3 \mathrm{D}$ reconstruction cut profiles.

tions and shapes of elements present in 3D cell culture, e.g. spheroids, branching and single cells, on a large and thus relevant volume (several $\mathrm{mm}^{3}$ ). Acquiring such information is of prior importance for the biologists in the realms of e.g. embryogenesis, angiogenesis and tumorigenesis. In particular the ability to detect isolated single cell distant from the main cell structure, since these cells may present a metastatic phenotype of interest. 